\title{
Langevin Poisson-Boltzmann equation: point-like ions and water dipoles near a charged surface
}

\author{
Ekaterina Gongadze ${ }^{1}$, Ursula van Rienen ${ }^{1}$, Veronika Kralj-Iglič² and Aleš Iglič ${ }^{3}$ \\ ${ }^{1}$ Institute of General Electrical Engineering, University of Rostock, Justus-von-Liebig Weg 2, D-18059 Rostock, Germany \\ ${ }^{2}$ Laboratory of Clinical Biophysics, Faculty of Medicine, University of Ljubljana, Lipičeva 2, SI-1000 Ljubljana, Slovenia \\ ${ }^{3}$ Laboratory of Biophysics, Faculty of Electrical Engineering, University of Ljubljana, Tržaška cesta 25, SI-1000 Ljubljana, \\ Slovenia
}

\begin{abstract}
Water ordering near a charged membrane surface is important for many biological processes such as binding of ligands to a membrane or transport of ions across it. In this work, the mean-field Poisson-Boltzmann theory for point-like ions, describing an electrolyte solution in contact with a planar charged surface, is modified by including the orientational ordering of water. Water molecules are considered as Langevin dipoles, while the number density of water is assumed to be constant everywhere in the electrolyte solution. It is shown that the dielectric permittivity of an electrolyte close to a charged surface is decreased due to the increased orientational ordering of water dipoles. The dielectric permittivity close to the charged surface is additionally decreased due to the finite size of ions and dipoles.
\end{abstract}

Key words: Electric double layer - Langevin Poisson-Boltzmann equation - Dielectric permittivity - Orientational ordering of water - Langevin-Bikerman equation

Abbreviations: EDL, electric double layer; PB, Poisson-Boltzmann; FEM, finite element method.

\section{Introduction}

The contact between a negatively charged surface and an electrolyte solution implies a particular ion rearrangement near the charged surface, i.e. the electrical double layer (EDL) (Helmholtz 1879; Gouy 1910; Chapman 1913; Stern 1924). The distribution of ions in the EDL can be described within the Poisson-Boltzmann (PB) theory (McLaughlin 1989; KraljIglič and Iglič 1996; Lamperski and Outhwaite 2002; Bivas 2006; Bivas and Ermakov 2007), expressing the competition between electrostatic interactions and the entropy of ions in the solution. Due to the electrostatic forces between the charged surface and ions in the solution, counter-ions are accumulated close to the charged surface, while coions are depleted from this region. As shown in the past, the properties of EDL may be influenced also by the ordering of water molecules in the region

Correspondence to: Aleš Iglič, Laboratory of Biophysics, Faculty of Electrical Engineering, University of Ljubljana, Tržaška cesta 25, SI-1000 Ljubljana, Slovenia

E-mail: ales.iglic@fe.uni-li.si of EDL (Gruen and Marčelja 1983; Cevc 1990; Israelachvili and Wernerstrom 1996; Butt et al. 2003; Manciu and Ruckenstein 2004; Arsov et al. 2009). This phenomenon is also subject of the present work. Most of the PB models of EDL (McLaughlin 1989; Cevc 1990; Lamperski and Outhwaite 2002) assume that the dielectric permittivity in the electrolyte is constant. In the absence of an explicit consideration of orientational ordering of water molecules, the assumption of constant permittivity is the consequence of the constant number of water molecules in PB theory. But actually, close to the charged surface the orientation of water molecules may result in a spatial variation of permittivity (Butt et al. 2003).

Outhwaite developed a modified PB theory of the electrical double layer composed of a mixture of hard spheres with point dipoles and finite sized ions (Outhwaite 1976, 1983). Later Kusalik and Patey (1988) and Torrie et al. (1989) implemented the reference hypernetted-chain theory for an EDL model of hard-sphere ions in a solvent composed of hard-spheres with point dipoles plus tetrahedral point quadrupoles. In this article, the PB mean-field theory for point-like ions is modified by taking into account the ori- 
entational ordering of water molecules. In the presented model, the dielectric permittivity is consistently related to the distribution of electric field strength and distribution of ions. The water molecules were modeled as Langevin dipoles (Outhwaite 1976, 1983; Coalson and Duncan 1996; Iglič et al. 2010), which is considered a very rough treatment of the dielectric properties of the solvent (Booth 1951).

The finite volume of ions (Bikerman 1942; Kralj-Iglič and Iglič 1996; Lamperski and Outhwaite 2002; Iglič et al. 2010) in the electrolyte solution (i.e. the excluded volume effect) is not taken into account. In accordance, the volume density of water is constant in the whole electrolyte solution (Kralj-Iglič and Iglič 1996).

\section{Theoretical predictions}

We consider a planar charged surface in contact with a solution of ions and water. The planar charged surface bears a charge characterized by a surface charge density $\sigma$. The Langevin dipole describes the water molecule with a non-zero dipole moment $(\boldsymbol{p})$. Recently, using the calculus of variation, the ion and water number density profiles corresponding to the minimum free energy (consisting of electrostatic field energy, translational entropy of ions and orientational entropy of water Langevin dipoles) of the system were calculated within a lattice-statistical model (Iglič et al. 2010):

$$
\begin{aligned}
& n_{+}(x)=n_{0} e^{-\Psi} \frac{n_{s}}{H} \\
& n_{-}(x)=n_{0} e^{\Psi} \frac{n_{s}}{H} \\
& n_{w}(x)=\frac{n_{0 w} n_{s}}{H} \frac{e_{0}}{p_{0}\left|\Psi^{\prime}\right|} \sinh \frac{p_{0}\left|\Psi^{\prime}\right|}{e_{0}}
\end{aligned}
$$

where the function $\mathrm{H}$, related to the finite size of ions, is given by:

$$
H(\phi, E)=2 n_{0} \cosh \Psi+\frac{e_{0} n_{0 w}}{p_{0}\left|\Psi^{\prime}\right|} \sinh \frac{p_{0}\left|\Psi^{\prime}\right|}{e_{0}}
$$

$n_{\mathrm{w}}(x)$ is the number density of water molecules, $n_{+}(x)$ and $n_{-}(x)$ are the number densities of counterions and coions, respectively, and

$$
\Psi(x)=\frac{e_{0} \phi(x)}{\mathrm{kT}}
$$

is the reduced electrostatic potential, $\phi(x)$ is the electrostatic potential, $E=k T\left|\Psi^{\prime}\right| / e_{0}$ is the magnitude of the electric field strength, $e_{0}$ is the elementary charge, $k T$ is the thermal energy, $n_{0}$ is the bulk number density of positively and negatively charged ions in electrolyte solution, $n_{0 \mathrm{w}}$ is the bulk number density of water, $n_{\mathrm{s}}$ being the number density

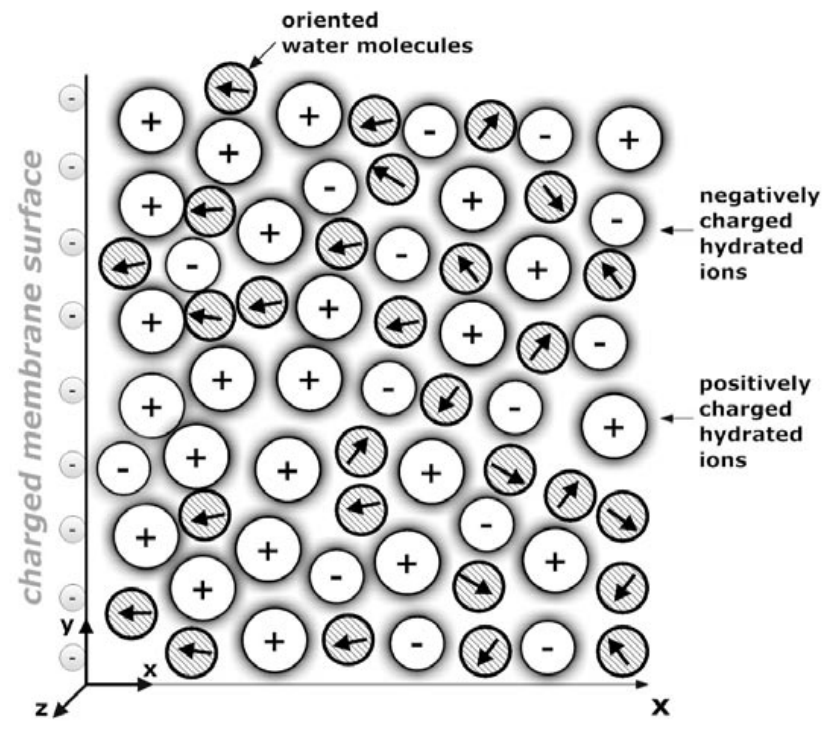

Figure 1. A schematic figure of an electrical double layer near a negatively charged planar membrane surface. The water molecules in the vicinity of charged surface are predominantly oriented towards the surface.

of lattice sites: $n_{\mathrm{S}}=n_{\mathrm{w}}(x)+n_{+}(x)+n_{-}(x)$, in bulk $n_{\mathrm{S}}=n_{0 \mathrm{w}}$ $+2 n_{0}, p_{0}$ is the magnitude of the water dipole moment and $\Psi^{\prime}$ is the first derivative of $\Psi$ with respect to $x$. The axis $x$ is perpendicular to the charged surface and points in the direction of the bulk solution (Fig. 1).

In the approximation of small electrostatic energy and small energy of Langevin dipoles in electric field compared to the thermal energy (relevant for point-like ions), i.e. $|\Psi|<1$ and $p_{0}\left|\Psi^{\prime}\right| / e_{0}<1$ we expand the functions $\cosh \Psi$ and $\sinh \left(p_{0}\left|\Psi^{\prime}\right| / e_{0}\right)$ in Eqs. (1-4) in Taylor series up to the first order: $\cosh \Psi \approx 1$ and $\sinh \left(p_{0}\left|\Psi^{\prime}\right| / e_{0}\right) \approx p_{0}\left|\Psi^{\prime}\right| / e_{0}$ to get the Boltzmann distribution functions for ions:

$$
n_{+}(x)=n_{0} e^{-\Psi}, n_{-}(x)=n_{0} e^{\Psi}
$$

and the constant number density of water molecules everywhere in the electrolyte solution:

$$
n_{w}(x)=n_{0 w}
$$

which is consistent with the classical PB theory for dimensionless (point-like) ions and constant permittivity (McLaughlin 1989; Cevc 1990; Kralj-Iglič and Iglič 1996; Lamperski and Outhwaite 2002; Bivas 2006). The charges of counterions, coions and water molecules (Langevin dipoles) contribute to the average microscopic volume charge density:

$$
\rho(x)=e_{0}\left(n_{+}(x)-n_{-}(x)\right)-\frac{\mathrm{d} P}{\mathrm{~d} x}
$$


The polarization $P$ is given by:

$$
P(x)=n_{0 w}\langle\mathbf{p}(x, \omega)\rangle_{B}
$$

where $\langle\mathbf{p}(x, \omega)\rangle_{\mathrm{B}}$ is the average of the dipole moment $\mathbf{p}$ over the angle distribution in thermal equilibrium. In the case of the negative surface charge densities $\sigma$, the value of $P(x)$ is considered negative since $\boldsymbol{P}$ points in the direction from the bulk to the charged surface, i.e. in the opposite direction to the direction of $x$-axis (see Fig. 1). The relative probability of finding a water dipole in an element of a solid angle $d \Omega=2 \pi \sin \omega d \omega$ is proportional to the Boltzmann factor $\exp \left(-W_{\mathrm{d}} / k T\right)$, where

$$
W_{d}=-\mathbf{p} \cdot \mathbf{E}=\mathbf{p} \cdot \nabla \phi=\left(k T / e_{0}\right) p_{0}\left|\Psi^{\prime}\right| \cos (\omega)
$$

is the energy of the water (Langevin) dipole $\boldsymbol{p}$ in the electric field $\mathbf{E}=-\nabla \phi$, $\omega$ is the angle between the dipole moment vector $\boldsymbol{p}$ and the vector $\nabla \phi$ Hence:

$$
\begin{aligned}
\langle\mathbf{p}(x, \omega)\rangle_{B} & =\frac{\int_{0}^{\pi} p_{0} \cos \omega \exp \left(-p_{0}\left|\Psi^{\prime}\right| \cos \omega / e_{0}\right) 2 \pi \sin \omega d \omega}{\int_{0}^{\pi} \exp \left(-p_{0}\left|\Psi^{\prime}\right| \cos \omega / e_{0}\right) 2 \pi \sin \omega d \omega} \\
& =-p_{0} \mathrm{~L}\left(\frac{p_{0}\left|\Psi^{\prime}\right|}{e_{0}}\right)
\end{aligned}
$$

The function $L(u)=(\operatorname{coth}(u)-1 / u)$ is the Langevin function. The Langevin function $L\left(p_{0}\left|\Psi^{\prime}\right| / e_{0}\right)$ describes the average magnitude of the Langevin dipole moments at given $x$. In our derivation we assumed an azimuthal symmetry. Inserting the Boltzmann distribution functions of ions (Eq. 6) and expression for polarization (Eqs. 9, 11):

$$
P(x)=n_{0 w}\langle\mathbf{p}(x, \omega)\rangle_{B}=-n_{0 w} p_{0} \mathrm{~L}\left(\frac{p_{0}\left|\Psi^{\prime}\right|}{e_{0}}\right)
$$

into Eq. (8), we get the expression for the average microscopic volume charge density in an electrolyte solution:

$$
\rho(x)=-2 e_{0} n_{0} \sinh \Psi+n_{0 w} p_{0} \frac{\mathrm{d}}{\mathrm{d} x}\left[\mathrm{~L}\left(p_{0}\left|\Psi^{\prime}\right| / e_{0}\right)\right]
$$

Inserting the above expression for volume charge density $\rho(x)$ (Eq. 13) into the Poisson equation:

$$
\phi^{\prime \prime}=-\frac{\rho}{\varepsilon_{0}}
$$

we get:

$$
\begin{aligned}
\phi^{\prime \prime}= & \frac{1}{\varepsilon_{0}}\left(2 e_{0} n_{0} \sinh \left(e_{0} \phi / \mathrm{kT}\right)-\right. \\
& \left.-n_{0 w} p_{0} \frac{\mathrm{d}}{\mathrm{dr}}\left[\mathrm{L}\left(p_{0} E / k T\right)\right]\right)
\end{aligned}
$$

where $\phi^{\prime \prime}$ is the second derivative of the electric potential $\phi$ with respect to $x, E=\left|\phi^{\prime}\right|$ is the magnitude of electric field strength, $\phi^{\prime}$ is the first derivative of the electric potential $\phi$ with respect to $x$ and $\varepsilon_{\mathrm{o}}$ is the permittivity of the free space (vacuum). The Langevin PB differential equation (Eq. 15) is subject to two boundary conditions. The first boundary condition is obtained by integrating the differential equation (Eq. 15):

$$
\phi^{\prime}(x=0)=-\frac{1}{\varepsilon_{0}}\left[\sigma+\left.n_{0 w} p_{0} \mathrm{~L}\left(p_{0} E / \mathrm{kT}\right)\right|_{x=0}\right]
$$

The condition, requiring electro-neutrality of the whole system, was taken into account in the derivation of Eq. (16). The second boundary condition is:

$$
\phi^{\prime}(x \rightarrow \infty)=0
$$

Based on Eq. (12), we can express the effective permittivity of the electrolyte solution $\left(\varepsilon_{\text {eff }}\right)$ in contact with the planar charged membrane surface as (see also Appendix):

$$
\varepsilon_{e f f}=1+\frac{|P|}{\varepsilon_{0} E}=1+n_{0 w} \frac{p_{0}}{\varepsilon_{0}} \frac{\mathrm{L}\left(p_{0} E / k T\right)}{E}
$$

Eqs. (15) and (16) can be rewritten in more general and condensed form as (see Appendix):

$$
\begin{aligned}
& \nabla \cdot\left(\varepsilon_{0} \varepsilon_{\text {eff }}(\mathbf{r}) \nabla \phi\right)=2 e_{0} n_{0} \sinh \left(e_{0} \phi / \mathrm{kT}\right) \\
& \nabla \phi\left(\mathbf{r}=\mathbf{r}_{\text {surf }}\right)=-\frac{\sigma \mathbf{n}}{\varepsilon_{0} \varepsilon_{\text {eff }}\left(\mathbf{r}=\mathbf{r}_{\text {surf }}\right)}
\end{aligned}
$$

where $\boldsymbol{r}$ is the position vector, $\boldsymbol{n}$ normal unit vector in the direction of $\nabla \phi$ and $\varepsilon_{e f f}(\boldsymbol{r})$ is defined by Eq. (18).

For small $p_{0} E / k T$ we can expand the Langevin function in Eq. (18) into Taylor series up to the cubic term $L(x) \approx x / 3$ $-x^{3} / 45$ to get:

$$
\varepsilon_{\text {eff }}=1+n_{0 w} p_{0}^{2} / 3 \mathrm{kT} \varepsilon_{0}-n_{0 w} p_{0}^{2}\left(p_{0} E / \mathrm{kT}\right)^{2} / 45 \mathrm{kT} \varepsilon_{0}
$$

It can be seen in Eq. (21) that $\varepsilon_{\text {eff }}$ decreases with increasing $E$. Since the value of $E$ decreases with increasing distance from the charged membrane surface (see for example

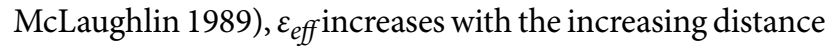
from the charged surface.

In this work Eq. (19) was solved numerically for a planar geometry using finite element method (FEM) within the program package Comsol Multiphysics 3.5a Software (Comsol $\mathrm{AB}$, Stockholm). The space dependency of $\varepsilon_{\text {eff }}$ (Eq. 18) in Eq. (19) was taken into account in an iterative procedure, where the initial value of $\varepsilon_{\text {eff }}$ is constant equal to the permittivity of the bulk solution. The boundary conditions (17) and (20) are taken into account. Fig. 2 shows the calculated spatial de- 
pendency of $\varepsilon_{\text {eff }}$ for two values of the surface charge density $\sigma$. The stronger decrease of $\varepsilon_{\text {eff }}$ near the charged surface for larger values of $\sigma$ is a consequence of stronger orientational ordering of water dipoles for larger values of $\sigma$.

In accordance with the results of other authors (Butt et al. 2003; Iglič et al. 2010), it can be concluded that due to the preferential orientation of water dipoles in the close vicinity of the charged membrane surface, the effective permittivity $\varepsilon_{\text {eff }}$ near the membrane surface is reduced relative to its bulk value. The decrease of $\varepsilon_{e f f}$ is more pronounced for larger values of $\sigma$ (Fig. 2).

\section{Discussion}

Recently, similar Langevin PB equation for point-like ions as given in Eq. (15) was derived using a statistical thermodynamics approach starting from a partition function of the system (Abrashkin et al. 2007). The Langevin PB equation for point-like ions, given in Abrashkin et al. (2007), can be derived also in somewhat different way by assuming the Boltzmann distribution function also for water (Langevin) dipoles:

$$
\begin{aligned}
& n_{+}(x)=n_{0} e^{-\Psi} \\
& n_{-}(x)=n_{0} e^{\Psi} \\
& n_{w}(x)=n_{0 w}\left\langle e^{-p_{0}\left|\Psi^{\prime}\right| \cos \omega / e_{0}}\right\rangle_{\omega}
\end{aligned}
$$

where

$$
\begin{aligned}
\left\langle e^{-p_{0}\left|\Psi^{\prime}\right| \cos \omega / e_{0}}\right\rangle_{\omega} & =\frac{2 \pi \int_{\pi}^{0} d(\cos \omega) e^{-p_{0}\left|\Psi^{\prime}\right| \cos \omega / e_{0}}}{4 \pi}= \\
& =\frac{e_{0}}{p_{0}\left|\Psi^{\prime}\right|} \sinh \frac{p_{0}\left|\Psi^{\prime}\right|}{e_{0}}
\end{aligned}
$$

Taking into account the Boltzmann distribution functions (Eqs. 22-24), a similar procedure as described above leads to the extension of the $\mathrm{PB}$ equation in the form as given in Abrashkin et al. (2007):

$$
\phi^{\prime \prime}=\frac{1}{\varepsilon_{0}}\left(2 e_{0} n_{0} \sinh \left(e_{0} \phi / \mathrm{kT}\right)-n_{0 w} p_{0} \frac{\mathrm{d}}{\mathrm{d} x}\left[F\left(p_{0} E / \mathrm{kT}\right)\right]\right)
$$

where the function $F$ is defined as:

$$
F(u)=\mathrm{L}(u) \frac{\sinh u}{u} .
$$

The corresponding effective permittivity $\left(\varepsilon_{\text {eff }}\right)$ is (Abrashkin et al. 2007):

$$
\varepsilon_{e f f}=1+n_{0 w} \frac{p_{0}}{\varepsilon_{0}} \frac{F\left(p_{0} E / k T\right)}{E}
$$

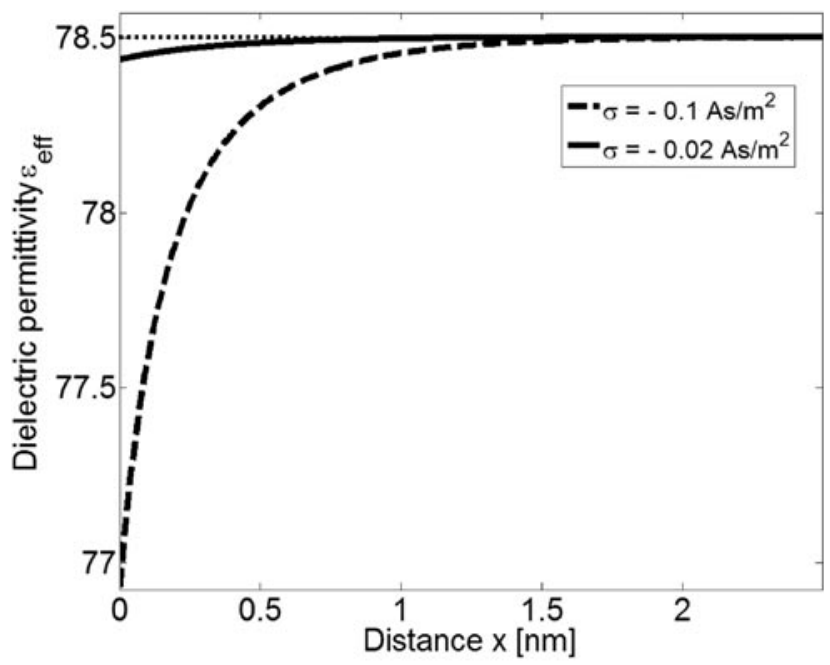

Figure 2. Effective dielectric permittivity $\varepsilon_{\text {eff }}$ as a function of the distance from the charged planar membrane surface for two values of the surface charge density $\sigma=-0.02 \mathrm{As} / \mathrm{m}^{2}$ and $\sigma=-0.1 \mathrm{As} / \mathrm{m}^{2}$ calculated by Langevin PB model for point-like ions. Eq. (19) is solved numerically for a planar geometry using FEM as described in the text. The dipole moment of water is $p_{0}=4.79 \mathrm{D}$, the bulk concentration of salt is $\mathrm{n}_{0} / N_{\mathrm{A}}=0.15 \mathrm{~mol} / \mathrm{l}$ and the bulk concentration of water $\mathrm{n}_{0 \mathrm{w}} / N_{\mathrm{A}}=55 \mathrm{~mol} / \mathrm{l}$.

For small $p_{0} E / k T$, we can expand the right hand side of Eq. (28) into Taylor series to get:

$$
\varepsilon_{\text {eff }}=1+n_{0 w} p_{0}^{2} / 3 \mathrm{kT} \varepsilon_{0}+n_{0 w} p_{0}^{2}\left(p_{0} E / \mathrm{kT}\right)^{2} / 30 k T \varepsilon_{0}
$$

It can be seen in Eq. (29) that $\varepsilon_{\text {eff }}$ increases with increasing $E$. Since the magnitude of electric field strength in electrolyte solution increases towards the charged membrane surface, Eqs. (28) and (29) predict the increase of $\varepsilon_{\text {eff }}$ in the vicinity of the charged membrane surface (Abrashkin et al. 2007). This is a consequence of the accumulation of water dipoles near the charged surface (due to an assumed Boltzmann distribution for water molecules) (see also Bazant et al. 2009), which prevails over the decrease of $\varepsilon_{\text {eff }}$ due to an increased orientational ordering of water molecules in a strong electric field as predicted by Eqs. (18) and (21) (Fig. 2).

Neglecting the finite volume of ions in Eqs. (28) and (29) reflects in the predicted increase of $\varepsilon_{\text {eff }}$ near the charged membrane surface (Abrashkin et al. 2007; Bazant et al. 2009, Iglič et al. 2010). The increase of $\varepsilon_{\text {eff }}$ near the charged membranme surface is not possible, if the excluded volume effect is taken into account, i.e. considering the accumulation of counterions near the charged membrane surface (Abrashkin et al. 2007). This actually prevents the accumulation of water molecules near a charged surface (Iglič et al. 2010). 


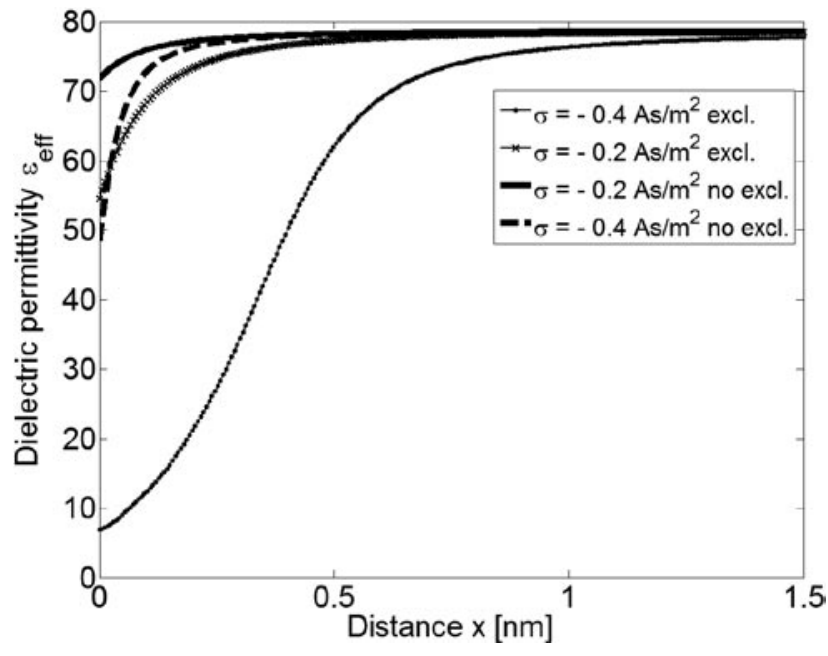

Figure 3. Effective dielectric permittivity $\varepsilon_{e f f}$ as a function of the distance from the charged planar membrane surface for two values of the surface charge density $\sigma=-0.2 \mathrm{As} / \mathrm{m}^{2}$ and $\sigma=-0.4 \mathrm{As} / \mathrm{m}^{2}$ calculated by Langevin PB model for pointlike ions (no excluded volume) and Langevin PB model with excluded volume. Eqs. (19) and (33) are solved numerically for a planar geometry using FEM as described in the text. The dipole moment of water is $p_{0}=4.79 \mathrm{D}$, the bulk concentration of salt is $\mathrm{n}_{0} / N_{\mathrm{A}}=0.15 \mathrm{~mol} / \mathrm{l}$ and the bulk concentration of water $\mathrm{n}_{0 \mathrm{w}} / N_{\mathrm{A}}=55 \mathrm{~mol} / \mathrm{l}$.

Considering the excluded volume effect within lattice statistical mechanics approach Eqs. (15) and (16) would be transformed into Langevin $\mathrm{PB}$ equation with excluded volume effect (Abrashkin et al. 2007; Iglič et al. 2010):

$$
\begin{aligned}
\phi^{\prime \prime}= & \frac{2 e_{0} n_{0} n_{s}}{\varepsilon_{0}} \frac{\sinh \left(e_{0} \phi / \mathrm{kT}\right)}{\mathrm{H}(\phi, E)}- \\
& -\frac{n_{0 w} n_{s} p_{0}}{\varepsilon_{0}} \frac{\mathrm{d}}{\mathrm{d} x}\left[\frac{F\left(p_{0} E / \mathrm{kT}\right)}{H(\phi, E)}\right]
\end{aligned}
$$

and

$$
\phi^{\prime}(x=0)=-\frac{1}{\varepsilon_{0}}\left[\sigma+\left.n_{0 w} n_{s} p_{0} \frac{\mathrm{L}\left(p_{0} E / \mathrm{kT}\right)}{H(\phi, E)}\right|_{x=0}\right]
$$

where

$$
H(\phi, E)=2 n_{0} \cosh \left(e_{0} \phi / \mathrm{kT}\right)+n_{0 w} \frac{\sinh \left(p_{0} E / \mathrm{kT}\right)}{\left(p_{0} E / \mathrm{kT}\right)}
$$

and $n_{\mathrm{s}}$ is the number density of lattice sites. Using the similar procedure as in Appendix, Eqs. (30) and (31) can be rewritten in more compact form of Langevin-Bikerman eq.:

$$
\nabla \cdot\left(\varepsilon_{0} \varepsilon_{\text {eff }}(\mathbf{r}) \nabla \phi\right)=-\rho_{\text {free }}(\mathbf{r})
$$

$$
\nabla \phi\left(\mathbf{r}=\mathbf{r}_{\text {surf }}\right)=-\frac{\sigma \mathbf{n}}{\varepsilon_{0} \varepsilon_{\text {eff }}\left(\mathbf{r}=\mathbf{r}_{\text {surf }}\right)}
$$

where $\rho_{\text {free }}(\boldsymbol{r})=e_{0}\left(n_{+}(x)-n_{-}(x)\right)$ is macroscopic (net) volume charge density in an electrolyte solution, i.e. the volume charge density of couterion and coions (for $n_{+}(\mathrm{x})$ and $n_{-}(\mathrm{x})$ see Iglič et al. 2010):

$$
\rho_{\text {free }}(\mathbf{r})=e_{0}\left(n_{+}(x)-n_{-}(x)\right)=-2 e_{0} n_{s} n_{0} \frac{\sinh \left(e_{0} \phi / \mathrm{kT}\right)}{H(\phi, E)}
$$

and $\varepsilon_{\text {eff }}(\boldsymbol{r})$ is the effective permittivity (Iglič et al. 2010):

$$
\varepsilon_{e f f}=1+n_{s} n_{0 w} \frac{p_{0}}{\varepsilon_{0}} \frac{F\left(p_{0} E / k T\right)}{H(\phi, E) E}
$$

Similary as above in the case of Eq. (19) also Eq. (33) was solved numerically for a planar geometry using the program package Comsol Multiphysics 3.5a Software (Fig. 3). The space dependency of $\varepsilon_{\text {eff }}$ in Eq. (33) was again taken into account in an iterative procedure. It can be seen in Fig. 3 that $\varepsilon_{\text {eff }}$ strongly decrese near the charged surface, the effect is being more pronounced for larger absolute values of $\sigma$. Assuming small electrostatic energies of ions and small energies of Langevin water dipoles, i.e. $\mathrm{e}_{0} \phi / k T<$ 1 and $p_{0} E / k T<1$ we can expand the general expression for the effective permittivity $\varepsilon_{\text {eff }}$ within Langevin PB theory with excluded volume effect (Eq. 36) into Taylor series to get (for $\left.n_{\mathrm{s}} \cong n_{0 \mathrm{w}}\right)$ :

$$
\begin{aligned}
\varepsilon_{e f f}= & 1+n_{0 w} p_{0}^{2} / 3 \mathrm{kT} \varepsilon_{0}-n_{0 w} p_{0}^{2}\left(p_{0} E / \mathrm{kT}\right)^{2} / 45 k T \varepsilon_{0}- \\
& -n_{0 w} p_{0}^{2}\left(e_{0} \phi / \mathrm{kT}\right)^{2} / 3 \mathrm{kT} \varepsilon_{0}
\end{aligned}
$$

It can be therefore concluded, that considering the excluded volume effect, the depletion of water dipoles near the charged surface due to accumulated counterions additionally decreases the dielectric permittivity of electrolyte solution near the charged surface (see also Fig. 3).

By comparison of the approximative expression for the effective permittivity $\varepsilon_{\text {eff }}$ calculated within Langevin PB theory taking into account the excluded volume effect (Eq. 37) and $\varepsilon_{e f f}$ within Langevin PB theory without considering the excluded volume effect (Eq. 21), we can see that the first three terms in expansions are equal in both models. The third term represents the effect of orientation of water molecules in the electric field near the charged membrane surface. The fourth term in Eq. (37) describes the decrease of $\varepsilon_{\text {eff near the charged membrane }}$ surface due to the depletion of water dipoles, because of accumulated counterions. Based on Eqs. (21) and (37), it can be concluded that the effective permittivity of the electrolyte $\varepsilon_{\text {eff }}$ near the charged membrane surface is reduced relative to its bulk value due to a preferential orientation of water molecules 
and depletion of water molecules in the close vicinity of the charged surface.

Note that unlike in Eq. (21), the third term in Eq. (29) does not agree with the third term in the general Eq. (37). We may, therefore, draw the conclusion that the assumption of the Boltzmann-like distribution functions for both, point-like ions and water molecules (Eqs. 22-24) is not consistent with the predictions of the Langevin PB theory for point-like ions presented in this work (Eqs. 18, 21; Fig. 2), and also not with the predictions of the Langevin PB theory with excluded volume effect (Eqs. 33-36; Fig. 3). For small $|\sigma|$ when the ions can be considered as pointlike, the ion distributions obey the Boltzmann functions (Eq. 6), while the number density of water molecules, $n_{\mathrm{w}}(x)$, is constant everywhere in the electrolyte solution (Eq. 7) (Kralj-Iglič and Iglič 1996). For larger $|\sigma|$, however, the Langevin PB theory with exclusion volume yields (Fig. 3) to a space variation of the water molecules density which is consistent with Fermi-Dirac-like ion distribution functions and not with Boltzmann distribution functions for point-like ions (Bikerman 1942; Kralj-Iglič and Iglič 1996; Iglič et al. 2010). Within Langevin-Bikerman theory with excluded volume, which considers the finite size of ions and water molecules, the water molecules are depleted in the region near the charged surface (and not accumulated as predicted by Eqs. (22-24)) due to the accumulation of counterions (Kralj-Iglič and Iglič 1996; Iglič et al. 2010).

To conclude: in this work, the PB theory for point-like ions was modified by introducing the orientational ordering of water molecules (Fig. 1). The water dipoles are described as Langevin dipoles with a given dipole moment. It is shown that the effective dielectric permittivity of the electrolyte solution $\varepsilon_{\text {eff }}$ decreases with increasing magnitude of the electric field strength (Eq. 21). Due to the increased magnitude of electric field in the vicinity of the charged surface in contact with an electrolyte solution, the effective permittivity $\varepsilon_{\text {eff }}$ of the electrolyte solution in the region near the charged membrane surface is decreased (Fig. 2). The predicted decrease of the permittivity relative to its bulk value is a consequence of the orientational ordering of water dipoles in the vicinity of the charged surface (Fig. 1). It can be also seen from Eq. (37) and Figs. 2 and 3 that for finite size ions, the dielectric permittivity profile $\varepsilon_{e f f}$ in the vicinity of a charged membrane surface is modulated by the depletion of water dipoles at the charged surface due to accumulated counterions (Iglič et al. 2010). The drop in the water number density near the charged surface results in additional decrease of permittivity (Fig. 3 and Eq. 37).

Acknowledgement. This work was supported by ARRS grants J39219-0381, P2-0232-1538 and DFG in the project A3 in Research Training Group 1505/1 “welisa”.

\section{Appendix}

Eq. (15) can be rewritten in a more general form as:

$$
\begin{aligned}
\nabla^{2} \phi(\mathbf{r})= & \frac{1}{\varepsilon_{0}}\left(2 e_{0} n_{0} \sinh \left(e_{0} \phi(\mathbf{r}) / \mathrm{kT}\right)-\right. \\
& \left.-n_{0 w} p_{0} \nabla \cdot\left[\mathbf{n L}\left(p_{0} E(\mathbf{r}) / k T\right)\right]\right)
\end{aligned}
$$

where $\mathbf{n}=\nabla \phi /|\nabla \phi|$ is the unit normal vector. By rearranging the terms we obtain from the above equation:

$$
\begin{aligned}
& \nabla \cdot\left(\varepsilon_{0} \nabla \phi\right)+n_{0 w} p_{0} \nabla \cdot\left[\mathbf{n L}\left(p_{0} E(\mathbf{r}) / k T\right)\right]= \\
& =2 e_{0} n_{0} \sinh \left(e_{0} \phi(\mathbf{r}) / \mathrm{kT}\right)
\end{aligned}
$$

and further

$$
\begin{aligned}
& \nabla \cdot\left[\varepsilon_{0} \nabla \phi+n_{0 w} p_{0} \mathbf{n L}\left(p_{0} E(\mathbf{r}) / k T\right)\right]= \\
& =2 e_{0} n_{0} \sinh \left(e_{0} \phi(\mathbf{r}) / \mathrm{kT}\right) \\
& \nabla \cdot\left[\varepsilon_{0}\left(1+\frac{n_{0 w} p_{0}}{\varepsilon_{0}} \frac{\mathbf{n}}{\nabla \phi} \mathrm{L}\left(p_{0} E(\mathbf{r}) / k T\right)\right) \nabla \phi\right]= \\
& =2 e_{0} n_{0} \sinh \left(e_{0} \phi(\mathbf{r}) / \mathrm{kT}\right) \\
& \nabla \cdot\left[\varepsilon_{0}\left(1+\frac{n_{0 w} p_{0}}{\varepsilon_{0}} \frac{1}{E} \mathrm{~L}\left(p_{0} E(\mathbf{r}) / k T\right)\right) \nabla \phi\right]= \\
& =2 e_{0} n_{0} \sinh \left(e_{0} \phi(\mathbf{r}) / \mathrm{kT}\right)
\end{aligned}
$$

where we took into account $\mathbf{n} / \nabla \phi=1 /|\nabla \phi|=1 / E$ and $\mathrm{E}=|\nabla \phi|$. Eq. (A5) can be further rewritten as:

$$
\nabla \cdot\left[\varepsilon_{0} \varepsilon_{\text {eff }}(\mathbf{r}) \nabla \phi(\mathbf{r})\right]=-\rho_{\text {free }}(\mathbf{r})
$$

where we defined $\varepsilon_{\text {eff }}(\mathbf{r})$ as the effective permittivity of an electrolyte solution:

$$
\varepsilon_{\text {eff }}(\mathbf{r})=1+n_{0 w} \frac{p_{0}}{\varepsilon_{0}} \frac{\mathrm{L}\left(p_{0} E(\mathbf{r}) / k T\right)}{E(\mathbf{r})}
$$

and

$$
\rho_{\text {free }}(\mathbf{r})=e_{0}\left(n_{+}(x)-n_{-}(x)\right)=-2 e_{0} n_{0} \sinh \left(e_{0} \phi(\mathbf{r}) / \mathrm{kT}\right)(\mathrm{A} 8)
$$

as the macroscopic (net) volume charge density in the electrolyte solution, i.e. the volume charge density of couterions and coions, where the number densities of counterions and coions $\left(n_{+}(x)\right.$ and $\left.n_{-}(x)\right)$ are given in Eq. (6). Also the boundary condition (16) can be transformed in more general and condensed form as:

$$
\begin{aligned}
\nabla \phi\left(\mathbf{r}=\mathbf{r}_{\text {surf }}\right)= & -\frac{1}{\varepsilon_{0}}[\sigma \mathbf{n}+ \\
& \left.+\left.\mathbf{n} n_{0 w} p_{0} \mathrm{~L}\left(p_{0} E(\mathbf{r}) / \mathrm{kT}\right)\right|_{\mathbf{r}=\text { surf }}\right]
\end{aligned}
$$




$$
\begin{aligned}
& \nabla \phi\left(\mathbf{r}=\mathbf{r}_{\text {surf }}\right)+\mathbf{n} \frac{n_{0 w} p_{0}}{\varepsilon_{0}} \mathrm{~L}\left(p_{0} E\left(\mathbf{r}=\mathbf{r}_{\text {surf }}\right) / \mathrm{kT}\right)= \\
& =-\frac{\sigma}{\varepsilon_{0}} \mathbf{n} \\
& \nabla \phi\left(\mathbf{r}=\mathbf{r}_{\text {surf }}\right)\left[1+\frac{\mathbf{n}}{\nabla \phi\left(\mathbf{r}=\mathbf{r}_{\text {surf }}\right)} \frac{n_{0 w} p_{0}}{\varepsilon_{0}}\right. \\
& \left.\mathrm{L}\left(p_{0} E\left(\mathbf{r}=\mathbf{r}_{\text {surf }}\right) / \mathrm{kT}\right)\right]=-\frac{\sigma}{\varepsilon_{0}} \mathbf{n} \\
& \nabla \phi\left(\mathbf{r}=\mathbf{r}_{\text {surf }}\right)\left[1+\frac{1}{E\left(\mathbf{r}=\mathbf{r}_{\text {surf }}\right)} \frac{n_{0 w} p_{0}}{\varepsilon_{0}}\right. \\
& \left.\mathrm{L}\left(p_{0} E\left(\mathbf{r}=\mathbf{r}_{\text {surf }}\right) / \mathrm{kT}\right)\right]=-\frac{\sigma}{\varepsilon_{0}} \mathbf{n}
\end{aligned}
$$

where we take into account $\mathbf{n} / \nabla \phi\left(\mathbf{r}=\mathbf{r}_{\text {surf }}\right)=1 / \mid \nabla \phi(\mathbf{r}=$ $\left.\mathbf{r}_{\text {surf }}\right) \mid=1 / E\left(\mathbf{r}=\mathbf{r}_{\text {surf }}\right)$.

By including the definition of the effective permittivity (Eq. A7), the above boundary condition (Eq. A12) takes the following form:

$$
\nabla \phi\left(\mathbf{r}=\mathbf{r}_{\text {surf }}\right) \varepsilon_{\text {eff }}\left(\mathbf{r}=\mathbf{r}_{\text {surf }}\right)=-\frac{\sigma}{\varepsilon_{0}} \mathbf{n}
$$

or

$$
\nabla \phi\left(\mathbf{r}=\mathbf{r}_{\text {surf }}\right)=-\frac{\sigma \mathbf{n}}{\varepsilon_{0} \varepsilon_{e f f}\left(\mathbf{r}=\mathbf{r}_{\text {surf }}\right)}
$$

\section{References}

Abrashkin A., Andelman D., Orland H. (2007): Dipolar PoissonBoltzmann equation: ions and dipoles close to charge surface. Phys. Rev. Lett. 99, 077801 doi:10.1103/PhysRevLett.99.077801

Arsov Z., Rappolt M., Grdodolnik J. (2009): Weakened hydrogen bonds in water confined between lipid bilayers: the existence of a long-range attractive hydration force. Chem. Phys. Chem. 10, 1438-1441 doi:10.1002/cphc.200900185

Bazant M. Z., Kilic M. S., Storey B., Ajdari A. (2009): Towards an understanding of induced-charge electrokinetics at large applied voltages in concentrated solutions. Adv. Colloid Interface Sci. 152, 48-88 doi:10.1016/j.cis.2009.10.001

Bikerman J. J. (1942): Structure and capacity of the electrical double layer. Phil. Mag. 33, 384-397

Bivas I. (2006): Electrostatic and mechanical properties of a flat lipid bilayer containing ionic lipids. Coll. Surf. A 282-283, 423-434 doi:10.1016/j.colsurfa.2005.12.010

Bivas I., Ermakov Y. A. (2007): Elasticity and electrostatics of amphiphilic layers. In: Advances in Planar Lipid Bilayers and
Liposomes. (Ed. A. Leitmannova Liu), Vol. 5, pp. 313-343, Elsevier, Amsterdam doi:10.1016/S1554-4516(06)05011-3

Booth F. (1951): The dielectric constant of water and the saturation effect. J. Chem. Phys. 19, 391-394 doi:10.1063/1.1748233

Butt H. J., Graf K., Kappl M. (2003): Physics and Chemistry of Interfaces. Wiley-VCH Verlag, Weinheim doi:10.1002/3527602313

Cevc G. (1990): Membrane electrostatics. Biochim. Biophys. Acta 1031, 311-382

Chapman D. L. (1913): A contribution to the theory of electrocapillarity. Philos. Mag. 25, 475-481

Coalson R. D., Duncan A. (1996): Statistical mechanics of a multipolar gas: a lattice field theory approach. J. Phys. Chem. 100, 2612-2620 doi:10.1021/jp952824m

Gouy M. G. (1910): Sur la constitution de la charge electrique a la surface d un electrolyte. J. Physique (France) 9, 457-468

Gruen D. W. R., Marčelja S. J. (1983): Spatially varying polarization in Water. Chem. Soc., Faraday Trans. II 79, 225-242

Helmholtz H. A. (1879): Studien über elektrische Grenzschichten. Ann. Phys. 7, 337-382

Israelachvili J. N., Wennerström H. (1996): Role of hydration and water structure in biological and colloidal interactions. Nature 379, 219-225 doi:10.1038/379219a0

Iglič A., Gongadze E., Bohinc K. (2010): Excluded volume effect and orientational ordering near charged surface in solution of ions and Langevin dipoles. Bioelectrochemistry 79, 223-227 doi:10.1016/j.bioelechem.2010.05.003

Kralj-Iglič V., Iglič A. (1996): A simple statistical mechanical approach to the free energy of the electric double layer including the excluded volume. J. Phys. II (France) 6, 477-491 doi:10.1051/jp2:1996193

Kusalik P. G., Patey G. N. (1988): On the molecular theory of aqueous electrolyte solution. II. Structural and thermodynamic properties of different models at infinite dilution. J. Chem. Phys. 89, 5843-5851

doi:10.1063/1.455535

Lamperski S., Outhwaite C. W. (2002): Volume term in the inhomogeneous Poisson-Boltzmann theory for high surface charge. Langmuir 18, 3423-3424 doi:10.1021/la011852v

Leland C., Allen L. C. (1974): Water. A Comprehensive Treatise. In: The Physics and Physical Chemistry of Water. (Ed. F. Franks), Vol. 1, Plenum, New York

Manciu M., Ruckenstein E. (2004): The polarization model for hydration/double layer interactions: the role of the electrolyte ions. Adv. Coll. Int. Sci. 112, 109-128 doi:10.1016/j.cis.2004.09.001

McLaughlin S. A. (1989): The electrostatic properties of membranes. Rev. Biophys. Chem. 18, 113-136 doi:10.1146/annurev.bb.18.060189.000553

Outhwaite C. W. (1976): A treatment of solvent effect in the potential theory of electrolyte solution. Mol. Phys. 31, 1345-1357 doi:10.1080/00268977600101061 
Outhwaite C. W. (1983): Towards a mean electrostatic potential treatment of an ion-dipole mixture or a dipolar system next to a plane wall. Mol. Phys. 48, 599-614

doi:10.1080/00268978300100431

Stern O. (1924): Zur Theorie der elektrolytischen Doppelschicht. Zeitschrift für Elektrochemie 30, 508-516

Torrie M., Kusalik P., Patey G.(1989): Molecular solvent model for an electrical double layer: reference hypernetted chain results for potassium chloride solutions. J. Chem. Phys. 90, $4513-4527$

doi:10.1063/1.456638

Received: January 17, 2011

Final version accepted: February 2, 2011 